OPEN ACCESS

Edited by:

Ren-You Gan

Institute of Urban Agriculture, Chinese

Academy of Agricultural Sciences

(CAAS), China

Reviewed by:

Libo Liu,

Northeast Agricultural

University, China

Nditange Shigwedha

Independent Researcher, Vevey,

Switzerland

*Correspondence:

Xia L

biology754@163.com

Hock Eng Khoo

2020153@glut.edu.cn

Specialty section:

This article was submitted to Nutrition and Microbes,

a section of the journal

Frontiers in Nutrition

Received: 17 September 2021 Accepted: 09 November 2021 Published: 03 December 2021

Citation:

Sun Y, Guan Y, Khoo HE and Li X (2021) In vitro Assessment of Chemical and Pre-biotic Properties of Carboxymethylated Polysaccharides From Passiflora edulis Peel, Xylan, and Citrus Pectin. Front. Nutr. 8:778563. doi: 10.3389/fnut.2021.778563

\section{In vitro Assessment of Chemical and Pre-biotic Properties of Carboxymethylated Polysaccharides From Passiflora edulis Peel, Xylan, and Citrus Pectin}

\author{
Yongjin Sun ${ }^{1}$, Yuan Guan ${ }^{1,2}$, Hock Eng Khoo ${ }^{1 *}$ and Xia Li ${ }^{1,2 *}$ \\ ${ }^{1}$ Department of Bioengineering, College of Chemistry and Bioengineering, Bioengineering Program, Guilin University of \\ Technology, Guilin, China, ${ }^{2}$ Guangxi Key Laboratory of Electrochemical and Magnetochemical Functional Materials, College \\ of Chemistry and Bioengineering, Bioengineering Program, Guilin University of Technology, Guilin, China
}

This study aimed to determine the carboxymethylation effect of crude water-soluble polysaccharides of Passiflora edulis peel (WPEP), xylan (XY), and citrus pectin (CP). Their chemical and pre-biotic properties were also determined. The polysaccharides were carboxymethylated by reacting with chloroacetic acid and sodium hydroxide. The carboxymethylated and non-carboxymethylated polysaccharides were also used as pre-biotics to study the growth pattern of selected intestinal microflora. These polysaccharides substituted the glucose solution in culture media for culturing Lactobacillus brevis GIM1.773, Lactobacillus plantarum GIM1.19, Lactobacillus delbrueckii subsp. bulgaricus GIM1.155, and Streptococcus thermophilus GIM1.540. The results showed that the carboxymethylated polysaccharides C-XY, C-CP, and c-WPEP, had substitution degrees of $0.682,0.437$, and 0.439 , respectively. The polysaccharides demonstrated resistance to digestion in the simulated human digestive models. The resistance to digestion was enhanced by carboxymethylation, especially the carboxymethylated CP and WPEP. The results also showed that the pre-biotic activities of the polysaccharides increased after carboxymethylation. The $\mathrm{c}-\mathrm{XY}$ had a better pre-biotic effect than $X Y$ and the other carbohydrate samples. The findings suggested that carboxymethylated polysaccharides may be developed into novel pre-biotics and nutraceuticals that could promote growth of the probiotic strains.

Keywords: chemical modification, growth curve, passion fruit, probiotic, functional group

\section{INTRODUCTION}

Pre-biotics are defined as substrates that could selectively promote growth and activity of the host microorganisms. They are non-digestible oligosaccharides that have a beneficial effect on the human gut. The substrates also maintain the balance of intestinal microecology $(1,2)$. These oligosaccharides are food for gut microflora, such as lactobacilli or bifidobacteria. These bacteria inhabit the human intestinal tract, and they are responsible for regulating fat storage and biosynthesis of essential vitamins (3). Pre-biotics are metabolized into lactic acid and short-chain fatty acids in the large intestine. These substances promote the growth of intestinal bacteria 
and improve the physiological health of the host. Regular consumption of pre-biotics is essential for maintaining good health and regulating intestinal microflora (4). Studies have shown that the experimental mice fed pre-biotics had improved intestinal microflora. Pre-biotics not only help to treat obesity but also improve the host's immune system and prevent the development of diseases like type 2 diabetes mellitus, irritable bowel syndrome, and colorectal cancer. These oligosaccharides can also indirectly regulate cardiovascular diseases (5).

Polysaccharides are potent sources of pre-biotics. They have antiviral, immuno-enhancing, hypoglycemic, antioxidation, and antitumor effects in addition to the pre-biotic properties (6-8). The substances also effectively promote the growth of intestinal microflora and increase short-chain fatty acid levels $(9,10)$. Literature showed that the polysaccharides extracted from the citrus peel (11), bamboo shoots (12), Ganoderma lucidum, and Poria cocos (13) exhibited pre-biotic potential. However, the pre-biotic activities of carboxymethylated polysaccharides were yet unknown. The consumption of sulfated polysaccharides from marine seaweeds as pre-biotics showed anti-inflammatory effects and prevented peptic-ulcer disease and gastrointestinal disorders (14). The disease-prevention mechanism is related to the blocking of the leucocyte adhered to the epithelium of blood vessels. The polysaccharides also prevented the migration of these cells to the inflammation sites. However, the biological activities of these polysaccharides are limited by their low solubility. Many scholars have also attempted to chemically modify the structures of polysaccharides to improve their physicochemical properties and bioactivities $(2,15)$.

The chemical modification of polysaccharides, especially carboxymethylation, has recently drawn wide attention. The carboxymethylation technique has been used to improve the physicochemical properties and bioactivity of plant polysaccharides. The carboxymethylated polysaccharides exhibited immunoregulatory, oxidation, and antitumor effects (16-19). A study on characterization of carboxymethylated xylan has been done previously, and the structural information has been obtained using ${ }^{13} \mathrm{C}$ nuclear magnetic resonance (20). The structural characteristics of carboxymethylated pectin had also been studied using Fourier-transform infrared spectroscopy (FTIR), X-ray diffraction, and thermogravimetric analysis (21). Moreover, physicochemical characteristics of polysaccharides extracted from passion fruit peel had been performed (22). As no previous study has been done on carboxymethylation of polysaccharides extracted from passion fruit peel, this study is, therefore, aimed to fill such a gap.

Currently, the valorization of natural resources and utilization of renewable energy resources leads to universal sustainability. The by-products of food processing are the new sources of sustainable food that are renewable and eco-friendly (23). Fruit peels or pericarps are some of the polysaccharide-rich wastes. Polysaccharides have been extracted from citrus and passion fruit peels. The use of polysaccharides from fruit peel provides a new idea for waste utilization. These polysaccharides are potent functional foods. Functional food is one of the most promising and fastest developing health foods in the food industry. Pre-biotics have been widely studied and commercially explored. The data on pre-biotic effects of carboxymethylated polysaccharides are also limited. Therefore, these polysaccharides have great application potential in the functional food and medical industries.

\section{MATERIALS AND METHODS}

\section{Chemicals and Reagents}

Fructooligosaccharide (FOS) was purchased from Guangdong Guanghua Chemical Factory Co., Ltd (Guangdong, China); trypsin, pancreatin solution, bile salt, and $\alpha$-amylase were purchased from Qingdao Hi-tech Park Haibo Biological Technology Co., Ltd. (China). All other chemicals and reagents were obtained from Xilong Chemical Co., Ltd. (Guangdong, China). All chemicals and reagents used in this study were of analytical grade.

\section{Sample Preparation}

The analytical grade of xylan (XY) was obtained from the Guangxi Institute of Botany (Guilin, China). The XY sample was prepared according to the method described by Miao et al. (24). Citrus pectin (PC) was purchased from the Shanghai Yuanye Biotechnology Co., Ltd. (Shanghai, China), and the WPEP sample was prepared according to the method described by Guan et al. (22). Lactobacillus brevis GIM1.773, L. plantarum GIM1.19, L. delbrueckii subsp. bulgaricus GIM1.155, and Streptococcus thermophilus GIM1.540 were obtained from the Guangdong Institute of Microbiology (Guangzhou, China).

\section{Preparation of Carboxymethylated Polysaccharides}

The chloroacetic acid-sodium hydroxide reaction procedure was adapted from the method described by Wang et al. (25). An exact $240 \mathrm{mg}$ of XY, CP, and WPEP was separately dissolved in $20 \mathrm{~mL}$ of $20 \%$ sodium hydroxide $(\mathrm{NaOH})$ solution and $50 \mathrm{~mL}$ isopropanol and stirred for $3 \mathrm{~h}$ in an ice-water bath to obtain a uniform suspension. Next, $6.0 \mathrm{~g}$ chloroacetic acid was mixed with $50 \mathrm{~mL}$ of isopropanol until complete dissolution was achieved. Then, $20 \mathrm{~mL}$ of $20 \% \mathrm{NaOH}$ solution was added to the mixture and heated for $3 \mathrm{~h}$ in a water bath of $60^{\circ} \mathrm{C}$. The conical flask was cooled to room temperature, and the solution $\mathrm{pH}$ was adjusted to 7 by adding $1 \mathrm{M}$ hydrochloric acid $(\mathrm{HCl})$. Finally, the mixture was dialyzed for $24 \mathrm{~h}$ with tap water, concentrated, and freeze-dried to obtain carboxymethylated polysaccharides. The carboxymethylated XY, CP, and WPEP were named c-XY, c-CP, and c-WPEP, respectively.

\section{Determination of Chemical Composition}

The phenol-sulfuric acid method was used to determine the total sugar content of the polysaccharide samples (26). Briefly, $1 \mathrm{~mL}$ of $0.1 \mathrm{mg} / \mathrm{mL}$ sample was added with $0.5 \mathrm{~mL}$ of $6 \%$ phenol reagent and $2.5 \mathrm{~mL}$ of concentrated sulfuric acid. The reacting solution was placed into a $\mathrm{HH}-\mathrm{W} 420$ water bath (Shanghai Fangrui Instrument Co., Ltd., Shanghai, China) at $100^{\circ} \mathrm{C}$ for $10 \mathrm{~min}$ and then cooled to room temperature. The changes in absorbances at $490 \mathrm{~nm}$ were determined, and the total sugar content was calculated based on the glucose standard curve $(0-1.0 \mathrm{mg} / \mathrm{mL})$. 
The m-hydroxydiphenyl method was used to determine the galacturonic acid content of the polysaccharide samples (27). In brief, $400 \mu \mathrm{L}$ sample solution $(0.1 \mathrm{mg} / \mathrm{mL})$ was added with sulfamic acid $(0.39 \mathrm{mg} / \mathrm{mL})$, homogenized, and then added with $2.5 \mathrm{~mL}$ of concentrated sulfuric acid. The mixture was placed in boiling water for $20 \mathrm{~min}$. After cooling to room temperature, $40 \mu \mathrm{L}$ of m-hydroxydiphenyl reagent was added to the solution mixture and kept at room temperature for $15 \mathrm{~min}$. The absorbance was measured at $595 \mathrm{~nm}$. The standard curve was plotted based on different concentrations of galacturonic acid (0-400 mg/mL).

Bradford method was used to determine the total protein content of the polysaccharide samples (28). Briefly, $1.0 \mathrm{~mL}$ of the sample solution $(0.1 \mathrm{mg} / \mathrm{mL})$ was added with $4 \mathrm{~mL}$ of Coomasie Blue reagent and then placed at room temperature for $5 \mathrm{~min}$. Bovine serum albumin $(0-1.0 \mathrm{mg} / \mathrm{mL})$ was used as the protein standard. The absorbance was measured at $595 \mathrm{~nm}$.

\section{Fourier-Transform Infrared Spectroscopy}

Exactly $1.0 \mathrm{mg}$ of the freeze-dried sample was mixed with $100 \mathrm{mg}$ potassium bromide, pulverized, and then pressed into disks. The Nicolet iS10 FTIR spectrometer (Thermo Scientific, Waltham, USA) was used to obtain the absorption spectra of compounds. The wavelengths used ranged from 4,000 to $400 \mathrm{~cm}^{-1}$.

\section{Scanning Electron Microscopy}

The polysaccharide samples $(1 \mathrm{mg} / \mathrm{mL})$ were dissolved in deionized water and freeze-dried to produce sample specimens. A SU5000 field emission scanning electron microscope (SEM) (Hitachi, Tokyo, Japan) was performed to observe the morphology of the polysaccharide samples at $20^{\circ} \mathrm{C}$ with an acceleration voltage of $5 \mathrm{kV}$ and magnification of $300 \times$.

\section{Determination of Degree of Substitution}

The degree of substitution (DS) was determined by the neutralization titration (29). Briefly, $10 \mathrm{mg}$ polysaccharide was diluted with $3 \mathrm{~mL} 70 \%$ methanol, then $10 \mathrm{~mL}$ distilled water and $5 \mathrm{~mL} 0.5 \mathrm{~mol} / \mathrm{L}$ sodium hydroxide were added, and finally titrated with $0.1 \mathrm{M} \mathrm{HCl}$ until the color of phenolphthalein in the mixture faded. The carboxymethylation degree (A) of the polysaccharide samples was determined as follows:

$$
\mathrm{A}=\frac{V_{0} M_{0}-\left(V_{2}-V_{1}\right) M}{W}
$$

where $\mathrm{V}_{0}$ is the amount of $\mathrm{NaOH}(\mathrm{mL})$ added, $\mathrm{V}_{1}$ is the volume of $\mathrm{HCl}$ used to titrate the sample $(\mathrm{mL}), \mathrm{V}_{2}$ is the amount of $\mathrm{HCl}$ $(\mathrm{mL})$ used, $\mathrm{M}_{0}$ is the increase in the concentration of sodium hydroxide $(0.5 \mathrm{~mol} / \mathrm{L}), \mathrm{M}$ is the concentration of $\mathrm{HCl}$ used to titrate the sample $(0.1 \mathrm{~mol} / \mathrm{L})$, and $\mathrm{W}$ is the mass of the sample (g). The degree of substitution (DS) was calculated as follows:

$$
D S=\frac{0.162 A}{1-0.058 A}
$$

\section{Hydrolysis Degree of Polysaccharides Simulated Saliva Digestion}

The digestion reagent of the simulated saliva was prepared by adding $0.764 \mathrm{~g}$ sodium chloride, $1.491 \mathrm{~g}$ potassium chloride, and
$0.133 \mathrm{~g}$ of calcium chloride into distilled water. The total volume was increased to $1,000 \mathrm{~mL}$, and the solution was adjusted to $\mathrm{pH}$ 6.9 with $1 \mathrm{M}$ sodium bicarbonate. An exact $0.345 \mathrm{~g} \alpha$-amylase was then dissolved with $400 \mathrm{~mL}$ of the digestion reagent, magnetically stirred for $20 \mathrm{~min}$, and finally filtered. The filtered was added with another $400 \mathrm{~mL}$ of the digestion reagent before adding $1 \mathrm{mg} / \mathrm{mL}$ sample solution at a ratio $(1: 1)$ and then placed in the water bath of $37^{\circ} \mathrm{C}$ to imitate the oral environment. The digesting samples were collected at $0 \mathrm{~h}$ and $0.5 \mathrm{~h}$ and then boiled for $5 \mathrm{~min}$ to inactivate the enzyme. The 3,5-dinitrosalicylic acid method and phenol-sulfuric acid were used to determine the reducing sugar and total sugar content. The degree of hydrolysis was calculated based on the formula as follows:

$$
\begin{aligned}
& \text { Hydrolysis degree }(\%) \\
& =\frac{\text { Hydrolyzed reducing sugar }}{\text { Total sugar }- \text { Non hydrolyzed reducing sugar }} \times 100
\end{aligned}
$$

\section{Simulated Gastric Digestion}

The experimental method was slightly modified from the method described previously (30-32). The buffer solution was prepared by adding $8.25 \mathrm{~g}$ disodium hydrogen phosphate monohydrate, $14.35 \mathrm{~g}$ monosodium phosphate, $8.0 \mathrm{~g}$ sodium chloride, $0.2 \mathrm{~g}$ potassium chloride, $0.1 \mathrm{~g}$ calcium chloride, and $0.18 \mathrm{~g}$ magnesium chloride hexahydrate with distilled water and diluted to $1,000 \mathrm{~mL}$. The $\mathrm{pH}$ of the buffer solution was adjusted to 1 , 2, or 3 with $1 \mathrm{M} \mathrm{HCl}$ solution. The sample weighing $100 \mathrm{mg}$ was added to $10.0 \mathrm{~mL}$ of the buffer solution and placed in a water bath at $37^{\circ} \mathrm{C}$ for $6 \mathrm{~h}$. An exact $1.0 \mathrm{~mL}$ of the sample solution was obtained at 4 and $6 \mathrm{~h}$ during the simulated gastric digestion to determine reducing sugar and total sugar content. The degree of hydrolysis was calculated using Equation (3).

\section{Simulated Small Intestinal Digestion}

The simulated small intestinal digestion was performed according to the method described previously $(2,31)$. The simulated small-intestinal juice was prepared by adding $5.40 \mathrm{~g}$ sodium chloride, $0.65 \mathrm{~g}$ potassium chloride, and $0.33 \mathrm{~g}$ calcium chloride into a conical flask, dissolved with distilled water, and diluted to $1,000 \mathrm{~mL}$. Next, $13 \mathrm{mg}$ trypsin, $100 \mathrm{~mL}$ of pancreatin solution $(7 \%, \mathrm{w} / \mathrm{w}), 200 \mathrm{~mL}$ of bile salt $(4 \%, \mathrm{w} / \mathrm{w})$, and the juice solution were mixed before the $\mathrm{pH}$ was adjusted to 7 with $1 \mathrm{M}$ sodium bicarbonate. Then, $1 \mathrm{mg} / \mathrm{mL}$ sample solution was mixed with the simulated small-intestinal juice at a ratio of $1: 1$. During the digestion, $1.0 \mathrm{~mL}$ of the digesting sample was separately collected at 4 and $6 \mathrm{~h}$ and then boiled for $5 \mathrm{~min}$ to inactivate the enzymes. The reducing sugar and total sugar content were determined. The degree of hydrolysis was calculated according to Equation (3).

\section{Preparation of Culture Media}

The basal medium for culturing $S$. thermophilus was prepared by mixing peptone $(5.0 \mathrm{~g})$, yeast extract powder $(10.0 \mathrm{~g})$, calcium carbonate $(1.0 \mathrm{~g})$, dipotassium phosphate $(2.0 \mathrm{~g})$, glucose $(15.0$ $\mathrm{g} / \mathrm{L})$, cysteine $(0.50 \mathrm{~g})$, and Tween $-80(1.0 \mathrm{~mL})$ in a beaker, and the mixture was heated until dissolution. The medium was topped 
up with distilled water to $1 \mathrm{~L}$. The $\mathrm{pH}$ was adjusted to 6.5 , and the solution was sterilized for $20 \mathrm{~min}$ at $121^{\circ} \mathrm{C}$. The basal medium for the other strains was prepared by mixing tryptone $(10 \mathrm{~g} / \mathrm{L})$, beef extract powder $(10.0 \mathrm{~g})$, yeast extract powder $(5.0 \mathrm{~g})$, ammonium citrate $(2.0 \mathrm{~g})$, dipotassium phosphate $(2.0 \mathrm{~g})$, manganese sulfate monohydrate $(0.30 \mathrm{~g})$, Tween-80 $(1 \mathrm{ml} / \mathrm{L})$, urea $(15 \mathrm{~g} / \mathrm{L})$, and sodium acetate $(5 \mathrm{~g} / \mathrm{L})$, and then heated until dissolution. The distilled water was then added to the mixture to obtain a total volume of $1 \mathrm{~L}$. The $\mathrm{pH}$ was adjusted to 6.5 , and the solution was sterilized. The experimental media were prepared by replacing glucose solution in the basal medium with XY, CP, WPEP, and the carboxymethylated polysaccharide samples.

\section{Pre-biotic Effect of the Carboxymethylated Polysaccharides}

The pre-biotic effect of the polysaccharide samples was determined based on the microbial growth assay. The polysaccharide samples were added to the culture media as the only carbon source. The four probiotic strains used were L. brevis, L. plantarum, L. delbrueckii subsp. bulgaricus, and $S$. thermophilus. Different concentrations of the polysaccharide samples were first used to screen for the microbial growthpromoting effect. In brief, a $100 \mu \mathrm{L}$ of probiotic solution $(2 \times$ $10^{8} \mathrm{CFU} / \mathrm{mL}$ ) was pipetted to the culture medium and then cultured for $48 \mathrm{~h}$ at $37^{\circ} \mathrm{C}$. The optical density (OD) values were determined by measuring the absorbance at a wavelength of $600 \mathrm{~nm}$. The OD value denotes the optimal concentration of polysaccharides used for the growth of the probiotic strains. The microbials were then cultured at different incubation times $(0$, $4,8,12,24$, and $36 \mathrm{~h}$ ) by applying the optimized polysaccharide concentration. The OD values of the cultures were measured, and the results were expressed as log $\mathrm{CFU} / \mathrm{mL}$. A linear regression equation was obtained for each probiotic strain. The optimal polysaccharide concentration of $3 \%(\mathrm{w} / \mathrm{v})$ was chosen as the only carbon source, and FOS was used for comparison. The growth curves of the four probiotic strains cultured using the culture media containing different polysaccharide samples were plotted.

\section{Statistical Analysis}

All data were expressed as mean \pm standard error of the mean $(\mathrm{n}=3)$. The statistically significant differences were determined between the different groups based on the analysis of variance coupled with Duncan's multiple range test and student's $t$-test. The statistical analysis was performed using SPSS 26.0 software. $P<0.05$ was considered a statistically significant difference.

\section{RESULTS AND DISCUSSION}

\section{Chemical Composition Analysis}

Three polysaccharides were used in carboxymethylation. They were XY, CP, and WPEP. The degrees of substitution for c-XY, c$\mathrm{CP}$, and c-WPEP were $0.68,0.44$, and 0.44 , respectively (Table 1 ). $\mathrm{XY}$ had the highest degree of saturation in comparison with $\mathrm{CP}$ and WPEP. The results also showed that the carboxymethylated polysaccharide samples had a significantly lower total sugar content than the non-carboxymethylated samples $(P<$ 0.05). Although the carboxymethylated polysaccharides had total protein content lesser than the non-carboxymethylated forms, no significant differences were found between the polysaccharide samples $(P>0.05)$. WPEP also had a significantly lower galacturonic acid content besides the total sugar and protein content. On the contrary, the c-XY and c-CP had a significantly higher galacturonic acid content than the non-carboxymethylated forms $(P<0.05)$.

The XY was a fine light-yellow powder, $C P$ was a white powder, and WPEP was a golden particle (Figure 1). The colors and appearances of XY, CP, and WPEP were remarkably changed after carboxymethylation. These changes indicated that the internal structure of the polysaccharides could have been modified chemically. Carboxymethylation of polysaccharides extracted from Cyclocarya paliurus showed a lower protein content than the non-carboxymethylated samples (33). Besides, the solubility of the polysaccharides increased after carboxymethylation (34). Moreover, the bioactivity of the c-XY improved (35).

The IR spectra of the c-XY and XY are shown in Figure 1A. The result showed that the broad peak at $3,480 \mathrm{~cm}^{-1}$ could be attributed to the stretching vibrations of the $\mathrm{OH}$ group. The IR peak at 2,930 $\mathrm{cm}^{-1}$ could also be attributed to the stretching vibration of the $\mathrm{CH}$ group. The result revealed $\mathrm{C}-\mathrm{O}-\mathrm{C}$ stretching vibrations at $1,330 \mathrm{~cm}^{-1}$. The peak spectra of c-XY close to 1,609 and $1,405 \mathrm{~cm}^{-1}$ also showed the characteristic absorption peaks of $\mathrm{C}=\mathrm{O}$ and $\mathrm{CO}$, respectively.

The IR spectra of the c-CP and CP are shown in Figure 1B. The broad peak at $3,420 \mathrm{~cm}^{-1}$ could be attributed to the stretching vibrations of the $\mathrm{OH}$ group. The IR peak at $2,926 \mathrm{~cm}^{-1}$ was due to the stretching vibration of the $\mathrm{CH}$ group in $\mathrm{c}-\mathrm{XY}$, and the peak at $1,330 \mathrm{~cm}^{-1}$ revealed $\mathrm{C}-\mathrm{O}-\mathrm{C}$ stretching vibrations of the carboxymethylated structure. The peak spectra of c-XY close to 1,616 and $1,419 \mathrm{~cm}^{-1}$ also showed the characteristic absorption peaks of $\mathrm{C}=\mathrm{O}$ and $\mathrm{CO}$, respectively.

The IR spectra of the c-WPEP and WPEP are shown in Figure 1C. The broad spectrum peak at $3,420 \mathrm{~cm}^{-1}$ could be attributed to the stretching vibrations of the $\mathrm{OH}$ group. Similar to $\mathrm{c}-\mathrm{XY}$ and $\mathrm{c}-\mathrm{CP}$, the $\mathrm{CH}, \mathrm{C}-\mathrm{O}-\mathrm{C}, \mathrm{C}=\mathrm{O}$, and $\mathrm{CO}$ stretching vibrations were found for the c-WPEP. Also, the spectra of XY, CP, WPEP, c-XY, c-CP, and c-WPEP indicated typical characteristic absorption peaks of the polysaccharides at wavelengths of 1,100 and $3,500 \mathrm{~cm}^{-1}$.

The carboxymethylated polysaccharides had a lower peak height (broad peak) than the non-carboxymethylated forms. There was a sharp band at $900 \mathrm{~cm}^{-1}$, where it arose from the $\mathrm{C} 1$ group frequency or ring frequency. It was the characteristic of $\beta$-glucosidic linkages between the sugar units. The peak spectra of c-XY, c-CP, and c-WPEP close to 1,600 and 1,425 $\mathrm{cm}^{-1}$ also revealed the characteristic absorption peaks of $\mathrm{C}=\mathrm{O}$ and CO, respectively. The findings indicated the successful carboxymethylation of the polysaccharide samples.

The carboxymethylation was performed using both aqueous and organic media. The use of organic medium has many advantages, including high reaction stability and degree of substitution. The substitution degree of XY was higher than $\mathrm{CP}$ and WPEP because the purity of XY was higher than these substances. CP and WPEP had the same substitution 
TABLE 1 | Chemical compositions of polysaccharide samples.

\begin{tabular}{|c|c|c|c|c|}
\hline Samples & Total sugar (\%) & Total protein (\%) & Galactoronic acid (\%) & DS \\
\hline WPEP & $54.23 \pm 1.62^{\mathrm{e}}$ & $2.98 \pm 0.43^{a}$ & $39.43 \pm 2.33^{a}$ & \\
\hline$X Y$ & $94.43 \pm 4.77^{\mathrm{a}}$ & $1.49 \pm 0.22^{\mathrm{c}}$ & $4.32 \pm 1.74^{f}$ & \\
\hline $\mathrm{CP}$ & $87.25 \pm 3.76^{b}$ & $1.33 \pm 0.92^{c}$ & $6.72 \pm 2.25^{\mathrm{e}}$ & \\
\hline c-WPEP & $39.46 \pm 1.25^{f}$ & $2.48 \pm 0.57^{b}$ & $33.23 \pm 1.92^{b}$ & $0.44 \pm 0.06^{b}$ \\
\hline$c-X Y$ & $79.12 \pm 3.62^{d}$ & $1.42 \pm 0.14^{c}$ & $9.43 \pm 1.43^{c}$ & $0.68 \pm 0.02^{a}$ \\
\hline $\mathrm{c}-\mathrm{CP}$ & $79.63 \pm 1.89^{c}$ & $1.12 \pm 0.91^{d}$ & $7.66 \pm 1.47^{d}$ & $0.44 \pm 0.04^{b}$ \\
\hline
\end{tabular}

Data are presented as mean \pm standard error of the mean of three replicates. Different lowercase superscript letters in the same column denote significant differences $(P<0.05)$.
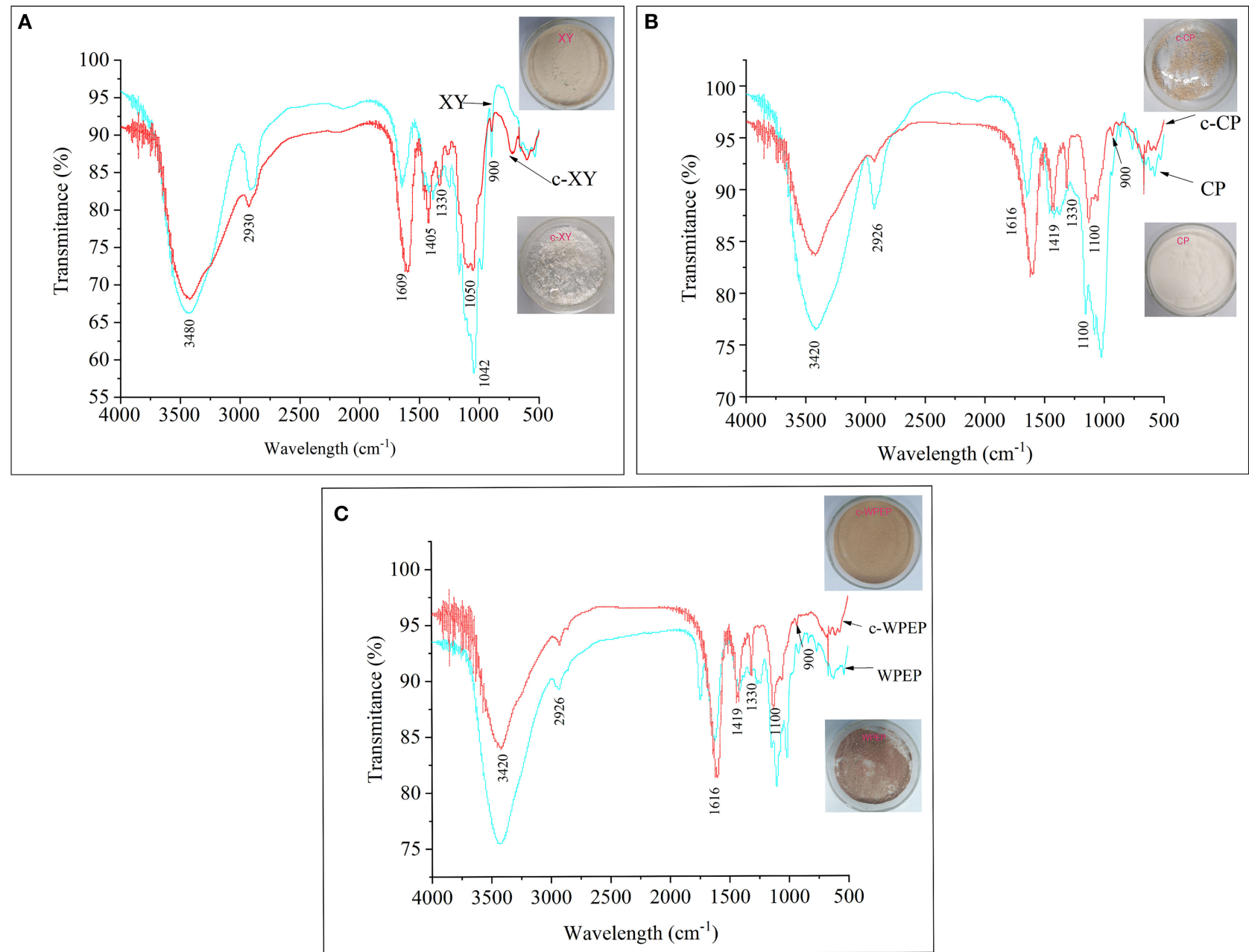

FIGURE 1 | FT-IR spectra of (A) xylan (XY), (B) citrus pectin (CP), and (C) crude water-soluble polysaccharides of Passiflora edulis peel (WPEP) samples.

degree because the main polysaccharide in WPEP was pectin. Literature shows that the purple passion fruit peel has as high as $12.6 \%$ of pectin. The major monosaccharides of pectin were rhamnose, arabinose, and galactose (36). These monosaccharides had been confirmed as the major components of the watersoluble polysaccharides of passion fruit peel (37).
The biological activity of polysaccharides is greatly affected by the functional groups that exist in the molecular structures. The presence of functional groups in a polysaccharide determines the size and bioactivity of the polysaccharide. Also, the chemical modification of a polysaccharide introduces new functional groups to its molecular structure. The spatial structure influences 


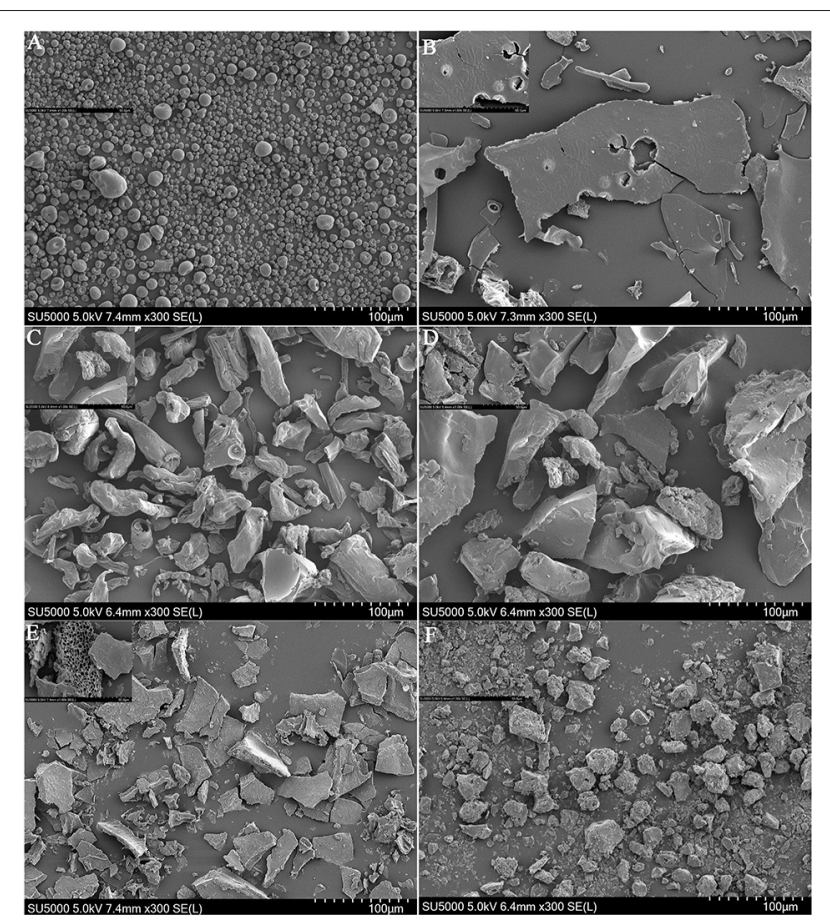

FIGURE 2 | SEM micrographs of (A) xylan (XY), (B) carboxymethylated xylan (C-XY), (C) citrus pectin (CP), (D) carboxymethylated citrus pectin (C-CP), (E) crude water soluble polysaccharides of Passiflora edulis peel (WPEP) samples, (F) carboxymethylated crude water soluble polysaccharides oPfassiflora edulis peel (c-WPEP) samples; magnification factor: $300 x$.

bioactivity of the polysaccharide. The polysaccharide structure with flexural waves has higher bioactivity than the others, whereas the polysaccharide with wrinkle-shaped or stretchable ribbons has low bioactivity (38).

The sweetness of a polysaccharide is derived from the monosaccharide molecules. The high number of hydroxyl groups $(-\mathrm{OH})$ of a polysaccharide denotes a high polysaccharide solubility in water and bioactivity (39). The sweetness of a polysaccharide is also attributed to the $\mathrm{OH}$ group. The carboxymethylation increased the intensity of stretching vibrations of the $\mathrm{OH}$ group. Therefore, the carboxymethylated polysaccharide could be sweeter than the non-carboxymethylated form.

\section{Scanning Electron Microscopy Analysis}

The surface structures of the polysaccharides were observed by SEM (Figure 2). The result showed that the surface structure of WPEP was multiporous, mildly rough, and unbounded. The surface structure of CP was less flaky, mild fibrous look, and some were rod-like structure; the $\mathrm{XY}$ had a granular shape. The changes in the surface structures of the polysaccharide samples were observed after the carboxymethylation, especially the surface of XY became flaky. The WPEP became lumpy after the carboxymethylation. The size of the flaky structure of c-WPEP was also reduced. The rodlike shape was not seen in the $\mathrm{c}-\mathrm{CP}$. The findings confirm that the structural surface of these polysaccharides had been modified by carboxymethylation.

The surface structural differences between the polysaccharide samples were due to the variation in molecular structures and bondings (40). The WPEP could contain other glycans besides pectin. The CP used in this study had $65 \%$ purity. The pectin in the purified extract might be bound together with glycans like xylan, xyloglucan, and glucuronoxylan (41). However, carboxymethylation increased the roughness and irregularity of the surface structure of xylan, with hollows and embossment (42). Also, CP prepared from manosonication assisted extraction formed an amorphous, rough, and hard surface with surface cracking and particles stuck to the surface.

\section{Hydrolysis Degree of Polysaccharides}

The study of resistance of the polysaccharide digestion was performed based on assays mimicking the human digestive tract. The digestion resistance was explored using simulated saliva, gastric juice, and small intestinal juice methods. The digestion resistance rate was determined based on the hydrolysis degree. The minimal differences among the polysaccharides and FOS in terms of the degree of hydrolysis are shown in Table 2. The results showed that most polysaccharide samples had a moderate degree of resistance to digestion. The hydrolysis degrees of c-WPEP assessed by the simulated saliva, gastric juice, and small intestinal juice methods were lower than the WPEP $(P<$ 0.05). It showed that the carboxymethylation of WPEP increased digestion resistance in the human digestive tract. The hydrolysis degrees of c-XY and c-CP were not significantly improved $(P>$ 0.05). The hydrolysis degrees of XY and CP after $4 \mathrm{~h}$ of hydrolysis using the simulated small intestinal juice test were significantly higher than the carboxymethylated samples $(P<0.05)$.

The minimal changes in hydrolysis degrees of the polysaccharide samples following digestion by saliva and gastric juice between 4 and $6 \mathrm{~h}$ indicated that the polysaccharide samples have a good resistance against digestion. Literature shows that $\mathrm{pH}$ values of gastric juice in a healthy individual ranged from 1.3 to 2.5 . The $\mathrm{pH}$ values increase to $4.5-5.8$ after eating. The ingested polysaccharides remain in the stomach for 4-6h, and the undigested substances enter the large intestine. The undigested polysaccharides are pre-biotics in the large intestine (37). The FTIR analysis showed that the molecular structures of the polysaccharide samples had a $\beta$-D glycosidic bond. The hydrolytic actions of human digestive enzymes on carbohydrates are mainly involved in the cleavage of $\alpha$-glycosidic bonds (43). Therefore, the polysaccharide samples were digestion resistant. In the simulated human digestive system, the degrees of hydrolysis of all polysaccharides were lower than $10 \%$. The polysaccharides also have relatively stable main structures. They are hydrolysis resistant, and they are potent pre-biotics (44).

Bile salts are the functional components of bile. They are biological surfactants involved in the digestion and absorption of lipids in the small intestine. The concentrations of bile salts in the small intestine also ranged between 4 and $20 \mathrm{mM}$. The values may fall to as low as $2.6 \mathrm{mM}$ in the fasted state or rise to over $15 \mathrm{mM}$ in the fed state $(45,46)$. The effects of different bile salts on the absorption of fluid, electrolytes, and monosaccharides 
TABLE 2 | Hydrolysis degrees (\%) of polysaccharides in simulated salivary, gastric, and intestinal conditions.

\begin{tabular}{|c|c|c|c|c|c|c|c|c|c|}
\hline \multirow[t]{3}{*}{ Samples } & \multirow{3}{*}{$\begin{array}{c}\text { Simulated } \\
\text { saliva } \\
0.5 \mathrm{~h}\end{array}$} & \multicolumn{6}{|c|}{ Simulated gastric } & \multicolumn{2}{|c|}{ Simulated intestinal } \\
\hline & & \multicolumn{3}{|c|}{$4 \mathrm{~h}$} & \multicolumn{3}{|c|}{$6 \mathrm{~h}$} & \multirow[t]{2}{*}{$4 \mathrm{~h}$} & \multirow[t]{2}{*}{$6 \mathrm{~h}$} \\
\hline & & pH 1 & pH 2 & pH 3 & pH 1 & pH 2 & pH 3 & & \\
\hline$X Y$ & $8.49 \pm 0.025^{c}$ & $2.00 \pm 0.014^{e}$ & $1.23 \pm 0.022^{\mathrm{e}}$ & $0.66 \pm 0.003^{9}$ & $4.07 \pm 0.004^{d}$ & $2.98 \pm 0.002^{d}$ & $3.53 \pm 0.002^{a}$ & $3.15 \pm 0.004^{c}$ & $3.5 \pm 0.026^{d}$ \\
\hline $\mathrm{CP}$ & $3.36 \pm 0.020^{f}$ & $2.13 \pm 0.030^{\circ}$ & $1.74 \pm 0.041^{c}$ & $2.15 \pm 0.069^{c}$ & $5.53 \pm 0.042^{b}$ & $3.51 \pm 0.041^{c}$ & $2.92 \pm 0.028^{c}$ & $3.36 \pm 0.02^{b}$ & $3.77 \pm 0.008^{c}$ \\
\hline WPEP & $10.30 \pm 0.023^{a}$ & $2.86 \pm 0.023^{b}$ & $3.89 \pm 0.045^{a}$ & $3.93 \pm 0.045^{a}$ & $2.34 \pm 0.025^{f}$ & $3.67 \pm 0.025^{b}$ & $1.87 \pm 0.010^{e}$ & $4.30 \pm 0.025^{a}$ & $4.70 \pm 0.020^{b}$ \\
\hline$c-X Y$ & $8.86 \pm 0.020^{b}$ & $1.49 \pm 0.005^{f}$ & $1.22 \pm 0.002^{\mathrm{e}}$ & $1.04 \pm 0.033^{\mathrm{e}}$ & $4.16 \pm 0.003^{c}$ & $2.38 \pm 0.001^{f}$ & $3.27 \pm 0.002^{b}$ & $0.88 \pm 0.006^{g}$ & $5.28 \pm 0.038^{a}$ \\
\hline $\mathrm{c}-\mathrm{CP}$ & $2.61 \pm 0.017^{9}$ & $2.04 \pm 0.004^{d}$ & $1.73 \pm 0.031^{c}$ & $2.58 \pm 0.02^{b}$ & $5.62 \pm 0.006^{a}$ & $4.11 \pm 0.032^{a}$ & $2.76 \pm 0.006^{d}$ & $1.09 \pm 0.009^{f}$ & $1.78 \pm 0.013^{9}$ \\
\hline c-WPEP & $7.70 \pm 0.024^{d}$ & $0.54 \pm 0.025^{9}$ & $1.31 \pm 0.023^{d}$ & $1.15 \pm 0.015^{d}$ & $1.14 \pm 0.025^{g}$ & $1.30 \pm 0.025^{9}$ & $1.22 \pm 0.0025^{f}$ & $1.50 \pm 0.004^{d}$ & $2.40 \pm 0.010^{f}$ \\
\hline FOS & $5.20 \pm 0.015^{\mathrm{e}}$ & $2.98 \pm 0.056^{a}$ & $2.64 \pm 0.073^{b}$ & $0.82 \pm 0.005^{\dagger}$ & $3.10 \pm 0.008^{e}$ & $2.50 \pm 0.002^{e}$ & $0.70 \pm 0.001^{\mathrm{g}}$ & $1.30 \pm 0.002^{\mathrm{e}}$ & $2.82 \pm 0.012^{\mathrm{e}}$ \\
\hline
\end{tabular}

Data are presented as mean \pm standard error of the mean of three replicates. Different lowercase superscript letters in the same column denote significant differences $(P<0.05)$.

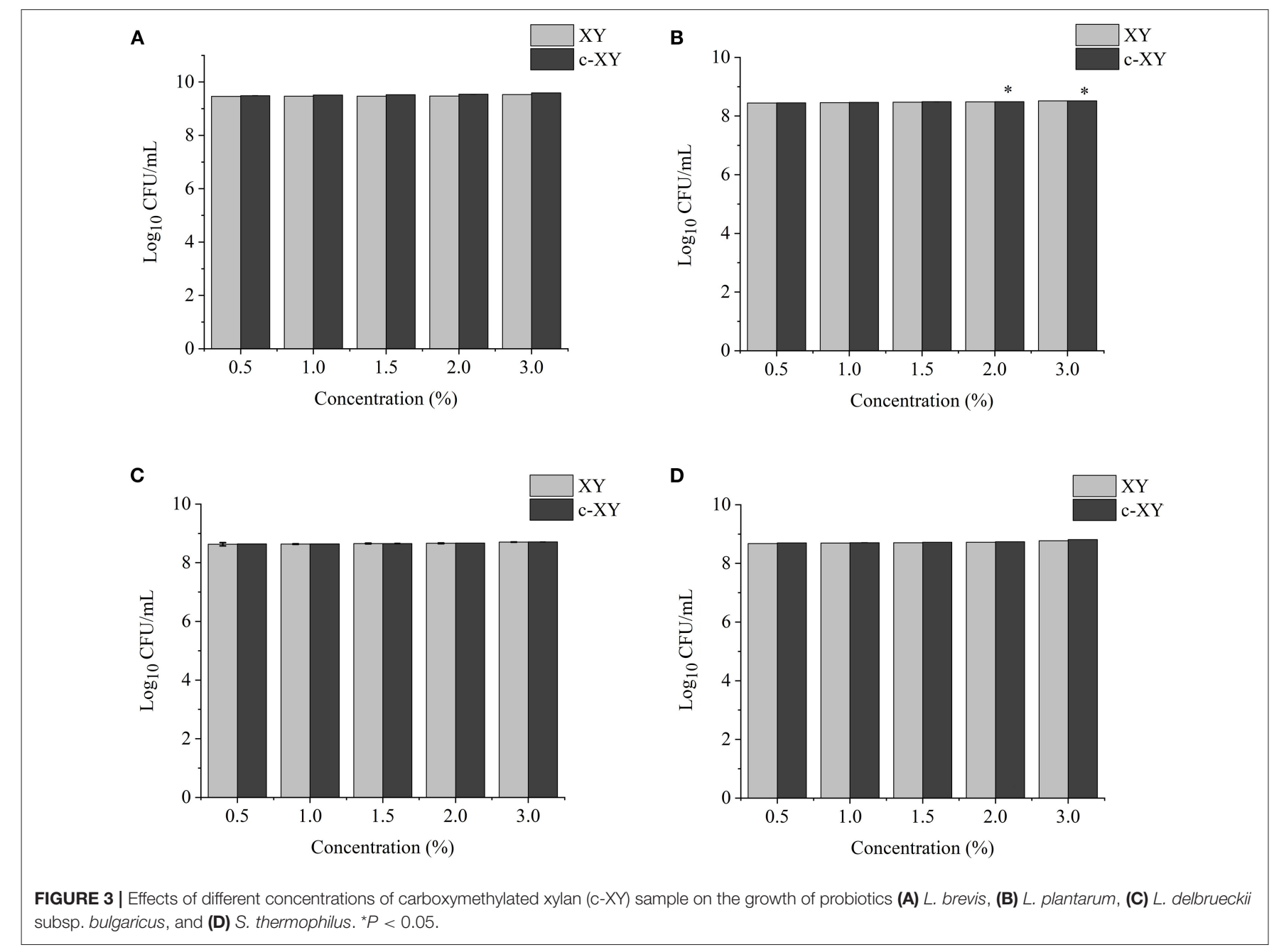

have been investigated in the small intestine of the experimental rats (47). The deoxycholate $(1 \mathrm{mM})$ impaired absorption of water and potassium in the jejunum, but not of sodium or glucose. At higher concentrations (2.5 and $5 \mathrm{mM}$ ), the secretion of fluid and electrolytes occurred, and glucose and fructose absorption was impaired (48). 

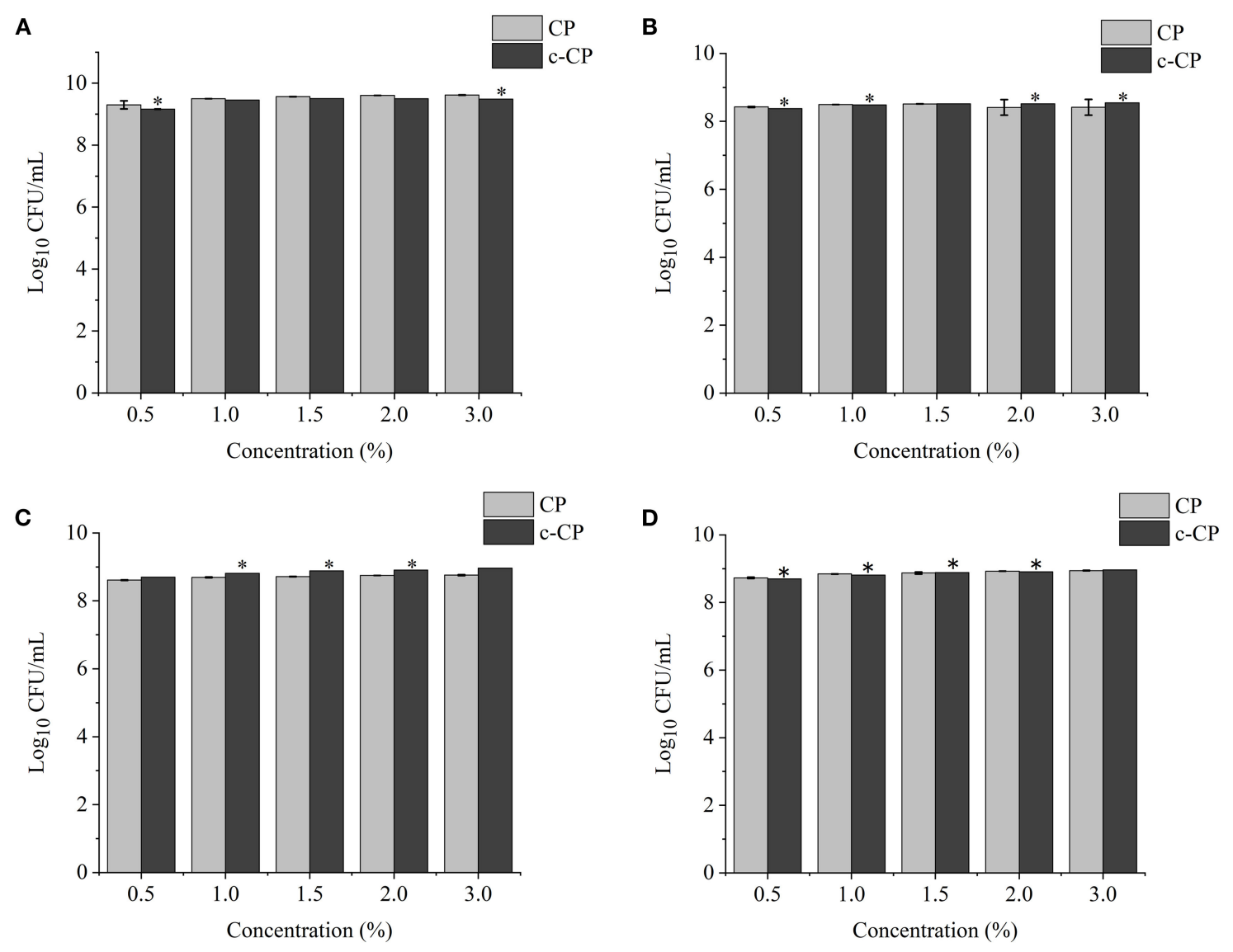

FIGURE 4 | Effects of different concentrations of carboxymethylated citrus pectin (c-CP) sample on the growth of (A) L. brevis, (B) L. plantarum, (C) L. delbrueckii subsp. bulgaricus, and (D) S. thermophilus. ${ }^{\star} P<0.05$.

\section{Effects of Different Concentrations of Polysaccharides on Probiotic Growth}

The effects of different concentrations of XY, CP, WPEP, c$\mathrm{XY}, \mathrm{c}-\mathrm{CP}$, and $\mathrm{c}$-WPEP on the growth of the probiotic strains were determined (Figures 3-5). The OD values reflected the microbial counts in the fermentation broth. The changes in the values represented the growth rate of the intestinal microflora. The growth of the probiotic strains could be more accurately expressed as CFU/mL (49). The regression equations of the standard curves for L. brevis, L. plantarum, L. delbrueckii subsp. bulgaricus, and $S$. thermophilus were $y=4^{-10} x+0.007\left(R^{2}=\right.$ 0.9995), $y=8^{-9} x-1.1215\left(R^{2}=0.9948\right), y=4^{-9} x-0.5944$ $\left(R^{2}=0.9926\right)$, and $y=2^{-9} x-0.26\left(R^{2}=0.9958\right)$, respectively. The results showed that c-XY and c-CP promoted the growth of the probiotic strains (Figures 3, 4), especially L. plantarum and L. delbrueckii subsp. bulgaricus. Hence, the c-WPEP inhibited the microbial growth (Figure 5).

The finding of this study revealed that $\mathrm{c}-\mathrm{XY}$ was the most effective pre-biotic in promoting the growth of L. brevis. It is because c-CP and c-WPEP significantly inhibited the growth of L. brevis and $S$. thermophilus. In this study, the polysaccharide samples promoted the growth of the Lactobacillus strains except for c-WPEP. The 3\% c-XY had the best effect in promoting the proliferation of the probiotic strains. As shown in Figures 3, 4, c$\mathrm{XY}$ and $\mathrm{c}-\mathrm{CP}$ at concentrations of up to $3 \%$ effectively increased the growth of L. plantarum $(P<0.05)$. On the contrary, some polysaccharides were not positively correlated with the growth of the probiotic strains.

Literature demonstrated that the effect of polysaccharides isolated from the dried root of Atractylodis macrocephalae (42) and Fu Brick tea (37) on the growth of probiotics was not concentration-dependent. When a higher concentration of the polysaccharides was used, the growth-promoting effect weakened. It could be due to the high sugar concentration causing an increase in the osmotic pressure and accumulation of metabolites, thus limiting the proliferation of bifidobacteria.

\section{Effect of Optimal Concentrations of Polysaccharides on Growth Curve of Probiotics}

The growth curves of the probiotic strains supplemented with the optimal concentrations of polysaccharide samples are presented in Figure 6. The addition of polysaccharide samples to the 

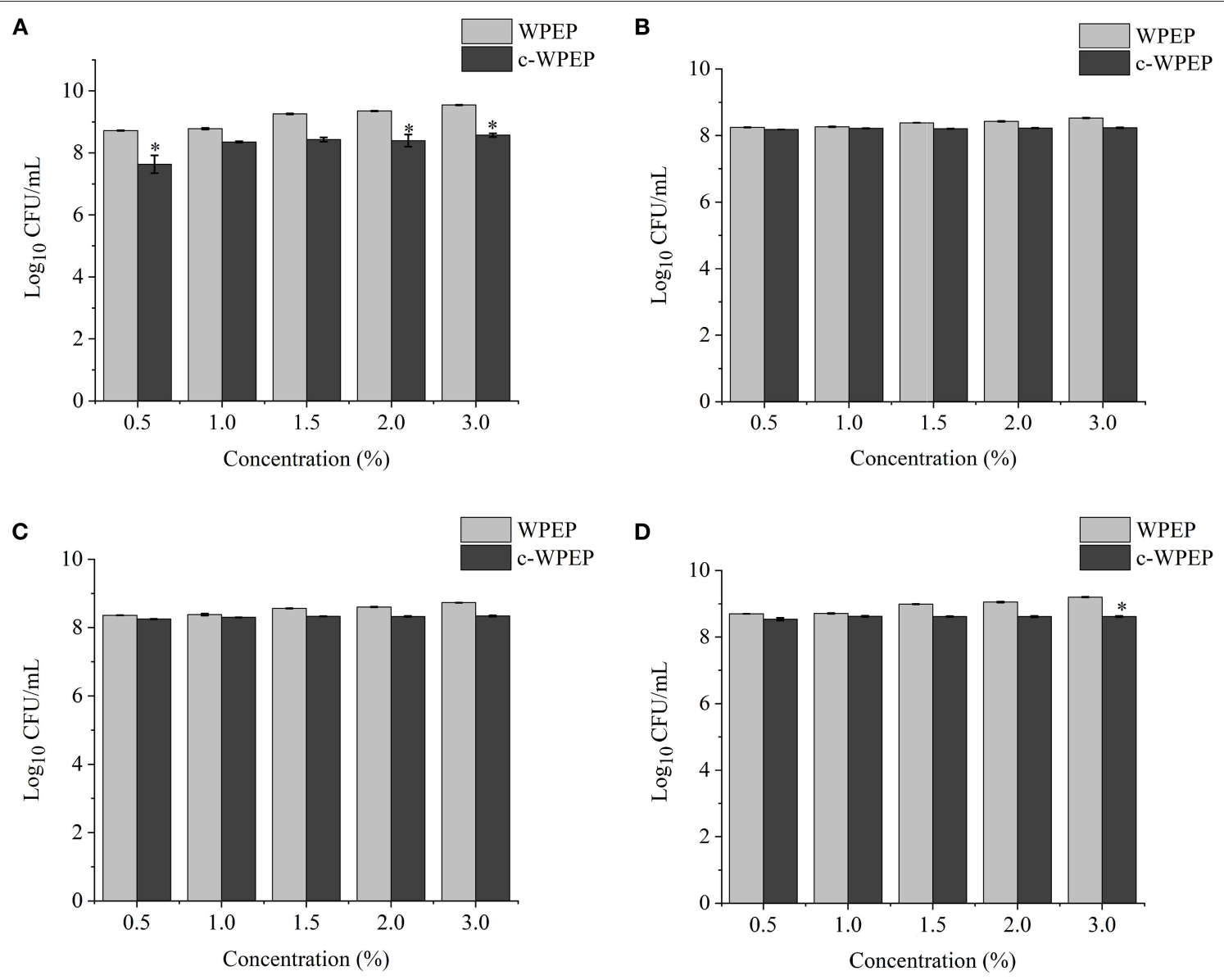

FIGURE 5 | Effects of different concentrations of the carboxymethylated crude water-soluble polysaccharides of Passiflora edulis peel (C-WPEP) sample on the growth of (A) L. brevis, (B) L. plantarum, (C) L. delbrueckii subsp. bulgaricus, and (D) S. thermophilus. ${ }^{\star} P<0.05$.

basal medium as sole carbon sources promoted microbial growth. The growth rate remained consistent for about $10 \mathrm{~h}$, and the growth rate increased rapidly and reached a maximum growth rate at $24 \mathrm{~h}$. After $24 \mathrm{~h}$, the growth rate started to drop gradually until the end of the experiment at $48 \mathrm{~h}$. The treatment with FOS showed the highest growth rates for all four probiotic strains.

The carboxymethylated polysaccharides significantly improved the growth performance of the probiotics compared with the non-carboxymethylated samples, especially for $L$. brevis. As shown in Figure 4, c-XY and c-CP had better growthpromoting effects than c-WPEP. Besides, the probiotics exposed to the carboxymethylated polysaccharides had a slightly stable growth phase after $24 \mathrm{~h}$ of incubation. Among the polysaccharides tested, c-XY was the most effective pre-biotic.

FOS is low molecular weight and low polymerization degree substance, and it has a better pre-biotic effect than the other polysaccharides. When FOS is added to the fermentation broth as the only energy source, it promotes the growth of the intestinal microflora. Literature also showed that FOS was the best carbon source for the proliferation of probiotics (12). Moreover, the ketone-rich FOS relieved allergic dermatitis by regulating the intestinal microflora, and it played an essential role in regulating the growth of these microorganisms (50).

The c-XY and FOS showed a similar growth performance of the four probiotics. This finding demonstrated that $\mathrm{c}-\mathrm{XY}$ is a more effective pre-biotic for promoting growth of the intestinal microflora than $\mathrm{c}-\mathrm{CP}$ and $\mathrm{c}$-WPEP. It could be because the carboxymethylation of XY changed in its surface structure and structural bonds. Previous studies showed that the characteristics of fructose-oligosaccharides, including structural units and degree of polymerization, had a fundamental influence on its probiotic activity; c-XY and FOS might also have similar chemical information.

The growth curves of the probiotics could be divided into different phases, such as the stagnation phase, logarithmic growth phase, stationary phase, and decline phase (51). S. thermophilus and $L$. brevis cultivated with most polysaccharide samples had a $4 \mathrm{~h}$ stagnation phase, whereas L. plantarum 

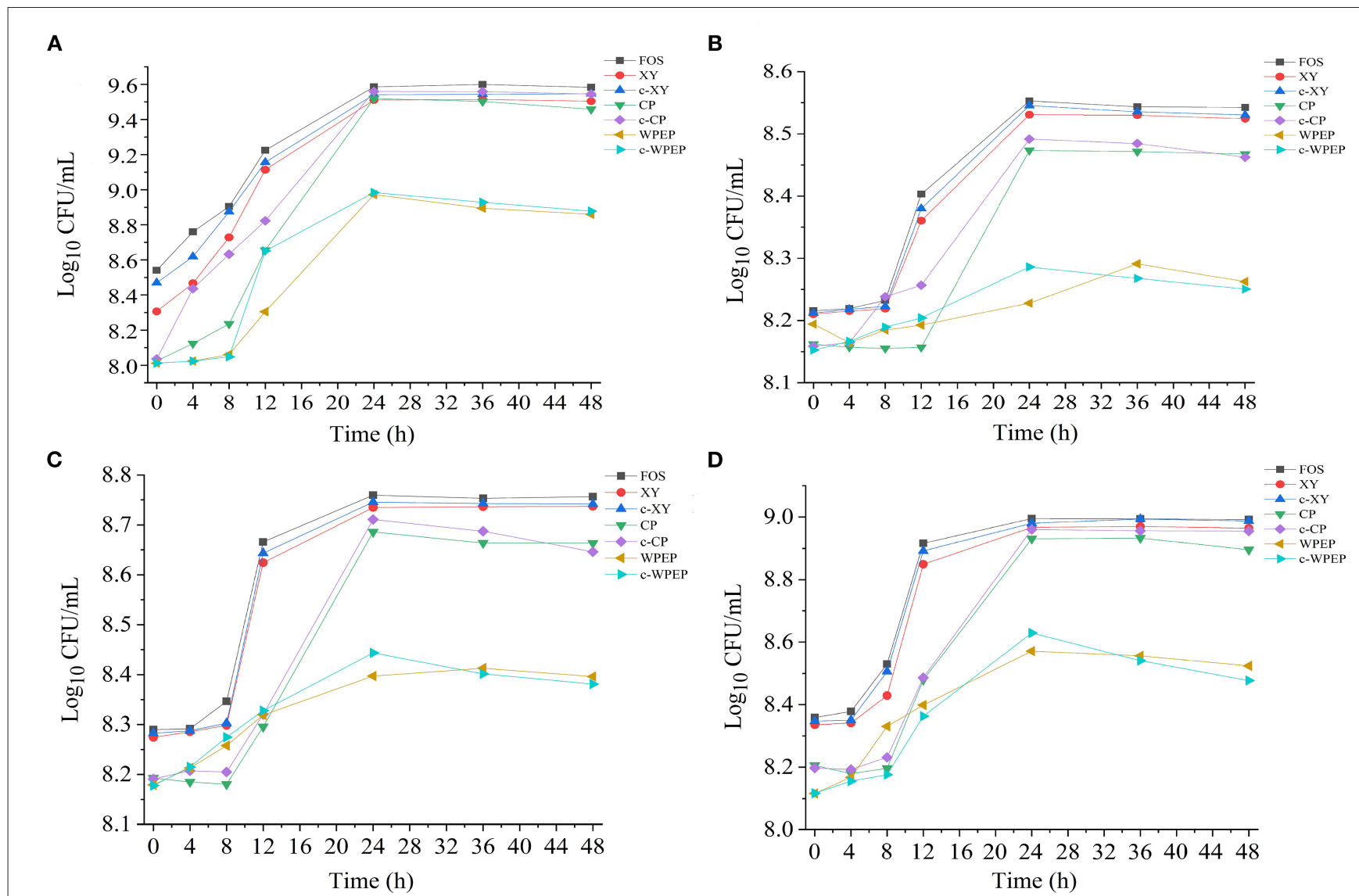

D

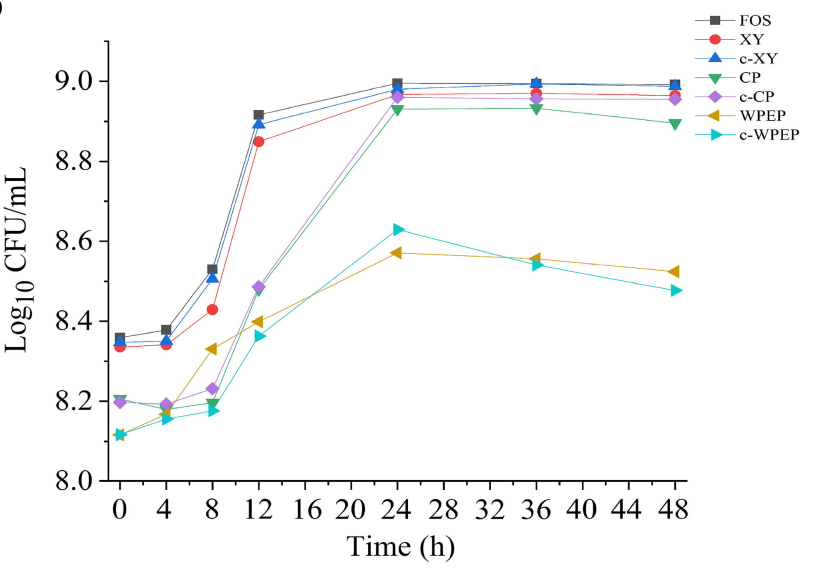

FIGURE 6 | Effects of polysaccharide samples on the growth of (A) L. brevis, (B) L. plantarum, (C) L. delbrueckii subsp. bulgaricus, and (D) S. thermophilus.

and L. delbrueckii subsp. bulgaricus showed a long hour of stagnation phase before entering the logarithmic growth phase. The declining growth curves were also observed if the probiotics were kept at a prolonged period, especially after $36 \mathrm{~h}$.

Studies have shown that the intestinal microflora mainly obtained nutrients from carbohydrate sources by digesting the complex polysaccharides (52). The c-WPEP showed a moderate effect in promoting the growth of the probiotics, but the result was less significant than the $\mathrm{c}-\mathrm{XY}$ and $\mathrm{c}$ CP. Among the three carboxymethylated polysaccharides, c-WPEP had the lowest sugar content. It indicated that c-WPEP did not provide enough energy for the growth of probiotics. Therefore, appropriate sources of prebiotics are needed for the optimal growth of intestinal microflora (53). Also, the solubility of the c-XY and c$\mathrm{CP}$ increased after carboxymethylation. The intestinal microflora could have fully utilized these carboxymethylated polysaccharides for their growth. As carboxymethylated polysaccharides have shorter chains than the noncarboxymethylated forms, the polysaccharides are easier to decompose and use by the intestinal microflora (54). Therefore, the carboxymethylated polysaccharides had a better pre-biotic effect.

\section{CONCLUSION}

The carboxymethylation of XY, CP, and WPEP was successfully performed using a combination of chloroacetic acid and $\mathrm{NaOH}$ reactions. The successful carboxymethylation of these polysaccharides was shown by the FTIR spectra. The carboxymethylated polysaccharides had total protein content lesser than the non-carboxymethylated forms, and no significant differences were found between the polysaccharide samples. The three carboxymethylated polysaccharides resisted hydrolysis based on the assays mimicking the human digestive tract, where c-WPEP had the best resistance to digestion. The hydrolysis degrees of c-WPEP accessed by the simulated saliva, gastric juice, and small intestinal juice methods were lower than the WPEP. It showed that the carboxymethylation of WPEP increased digestion resistance in the human digestive tract. The uses of c-XY, c-CP, and c-WPEP as sole carbon sources demonstrated a variation in the effects on the growth of L. brevis, L. plantarum, L. delbrueckii subsp. bulgaricus, and S. thermophilus. These probiotic strains had different abilities to decompose and utilize the polysaccharides. The carboxymethylated polysaccharide samples also demonstrated better pre-biotic effects than the non-carboxymethylated samples, and c-XY had a better prebiotic-promoting effect than $\mathrm{c}-\mathrm{CP}$ and $\mathrm{c}$-WPEP. The findings 
collectively suggested that $\mathrm{c}-\mathrm{XY}, \mathrm{c}-\mathrm{CP}$, and $\mathrm{c}$-WPEP are potent pre-biotics that should be developed into dietary supplements for regulating Lactobacillus and Streptococcus in the human gut.

\section{DATA AVAILABILITY STATEMENT}

The original contributions presented in the study are included in the article/Supplementary Material, further inquiries can be directed to the corresponding author.

\section{AUTHOR CONTRIBUTIONS}

YS: conceptualization, investigation, methodology, software, and writing-original draft. HEK: supervision, formal analysis, writing-review, and editing. YG: methodology, project administration, and funding acquisition. XL: supervision, project administration, funding acquisition, writing-review, and editing. All authors contributed to the article and approved the submitted version.

\section{REFERENCES}

1. Gibson GR, Hutkins R, Sanders ME, Prescott SL, Reimer RA, Salminen SJ, et al. The International Scientific Association for Probiotics and Prebiotics (ISAPP) consensus statement on the definition and scope of prebiotics. Nat Rev Gastroenterol Hepatol. (2017) 14:491-502. doi: 10.1038/nrgastro.2017.75

2. Li F, Sun X, Yu W, Shi C, Zhang X, Yu H, et al. Enhanced konjac glucomannan hydrolysis by lytic polysaccharide monooxygenases and generating prebiotic oligosaccharides. Carbohydr Polym. (2021) 253:117241. doi: 10.1016/j.carbpol.2020.117241

3. Gurpilhares DD, Cinelli LP, Simas NK, Pessoa A, Sette LD. Marine prebiotics: polysaccharides and oligosaccharides obtained by using microbial enzymes. Food Chem. (2019) 280:175-86. doi: 10.1016/j.foodchem.2018.12.023

4. Hill C, Guarner F, Reid G, Gibson GR, Merenstein DJ, Pot B, et al. The International Scientific Association for Probiotics and Prebiotics consensus statement on the scope and appropriate use of the term probiotic. Nat Rev Gastroenterol Hepatol. (2014) 11:506-14. doi: 10.1038/nrgastro.2014.66

5. Moludi J, Maleki V, Jafari-Vayghyan H, Vaghef-Mehrabany E, Alizadeh M. Metabolic endotoxemia and cardiovascular disease: a systematic review about potential roles of prebiotics and probiotics. Clin Exp Pharmacol Physiol. (2020) 47:927-39. doi: 10.1111/1440-1681.13250

6. Huang C, Zhu Z, Cao X, Chen X, Fu Y, Chen Z, et al. A pectic polysaccharide from Sijunzi decoction promotes the antioxidant defenses of SW480 cells. Molecules. (2017) 22:1341. doi: 10.3390/molecules22081341

7. Kong X, Duan W, Li D, Tang X, Duan Z. Effects of polysaccharides from Auricularia auricula on the immuno-timulatory activity and gut microbiota in immunosuppressed mice induced by cyclophosphamide. Front Immunol. (2020) 11:595700. doi: 10.3389/fimmu.2020.595700

8. Tong C, Chen Z, Liu F, Qiao Y, Chen T, Wang X. Antiviral activities of Radix isatidis polysaccharide against pseudorabies virus in swine testicle cells. BMC Complement Med Ther. (2020) 20:48. doi: 10.1186/s12906-020-2838-4

9. Kim M, Qie YQ, Park J, Kim CH. Gut microbial metabolites fuel host antibody responses. Cell Host Microbe. (2016) 20:202-14. doi: 10.1016/j.chom.2016.07.001

10. Xu J, Wang $\mathrm{R}$, Zhang $\mathrm{H}, \mathrm{Wu}$ J, Zhu L, Zhan X. In vitro assessment of prebiotic properties of oligosaccharides derived from four microbial polysaccharides. LWT-Food Sci Technol. (2021) 147:111544. doi: 10.1016/j.lwt.2021.1 11544

11. Zhang S, Hu H, Wang L, Liu F, Pan S. Preparation and prebiotic potential of pectin oligosaccharides obtained from citrus peel pectin. Food Chem. (2018) 244:232-7. doi: 10.1016/j.foodchem.2017.10.071

\section{FUNDING}

This study was supported by the National Natural Science Foundation of China (31860251), the Guangxi Science \& Technology Program (AD20297088), and the Guangxi Key Laboratory of Electrochemical and Magnetochemical Functional Materials (EMFM20211104 and EMFM20211102).

\section{ACKNOWLEDGMENTS}

All authors would like to thank Guozhu Zhang and Miao Zhang for helping in part of this research work.

\section{SUPPLEMENTARY MATERIAL}

The Supplementary Material for this article can be found online at: https://www.frontiersin.org/articles/10.3389/fnut.2021. 778563/full\#supplementary-material
12. He S, Wang $\mathrm{X}$, Zhang $\mathrm{Y}$, Wang J, Sun $\mathrm{H}$, Wang J, et al. Isolation and prebiotic activity of water-soluble polysaccharides fractions from the bamboo shoots (Phyllostachys praecox). Carbohydr Polym. (2016) 151:295304. doi: 10.1016/j.carbpol.2016.05.072

13. Khan I, Huang G, Li X, Leong W, Xia W, Hsiao WLW. Mushroom polysaccharides from Ganoderma lucidum and Poria cocos reveal prebiotic functions. J Funct Foods. (2018) 41:191-201. doi: 10.1016/j.jff.2017.12.046

14. Zaporozhets TS, Besednova NN, Kuznetsova TA, Zvyagintseva TN, Makarenkova ID, Kryzhanovsky SP, et al. The prebiotic potential of polysaccharides and extracts of seaweeds. Russ J Mar Biol. (2014) 40:1-9. doi: 10.1134/S1063074014010106

15. Huang G, Chen X, Huang H. Chemical modifications and biological activities of polysaccharides. Curr Drug Targets. (2016) 17:1799-803. doi: 10.2174/1389450117666160502151004

16. Chen F, Huang G. Preparation and immunological activity of polysaccharides and their derivatives. Int J Biol Macromol. (2018) 112:211-6. doi: 10.1016/j.ijbiomac.2018.01.169

17. Li XL, Tu XF, Thakur K, Zhang YS, Zhu DY, Zhang JG, et al. Effects of different chemical modifications on the antioxidant activities of polysaccharides sequentially extracted from peony seed dreg. Int J Biol Macromol. (2018) 112:675-85. doi: 10.1016/j.ijbiomac.2018.01.216

18. Liu Y, You Y, Li Y, Zhang L, Yin L, Shen Y, et al. The characterization, selenylation and antidiabetic activity of mycelial polysaccharides from Catathelasma ventricosum. Carbohydr Polym. (2017) 174:72-81. doi: 10.1016/j.carbpol.2017.06.050

19. Ming K, Chen Y, Shi J, Yang J, Yao F, Du H, et al. Effects of Chrysanthemum indicum polysaccharide and its phosphate on anti-duck hepatitis a virus and alleviating hepatic injury. Int J Biol Macromol. (2017) 102:813-21. doi: 10.1016/j.ijbiomac.2017.04.093

20. Wang L, Qin X, Miao X, Chen H, Zhou Y, Cai A. Synthesis and nondestructive detailed structure characterization of carboxymethyl xylan from bagasse. $J$ Carbohydr Chem. (2020) 39:131-44. doi: 10.1080/07328303.2020.1748643

21. Muthukumaran C, Kanmani BR, Sharmila G, Kumar NM, Shanmugaprakash M. Carboxymethylation of pectin: optimization, characterization and in-vitro drug release studies. Carbohydr Polym. (2018) 194:311-8. doi: 10.1016/j.carbpol.2018.04.042

22. Guan Y, Sun H, Chen H, Li P, Shan Y, Li X. Physicochemical characterization and the hypoglycemia effects of polysaccharide isolated from Passiflora edulis Sims peel. Food Funct. (2021) 12:4221-30. doi: 10.1039/D0FO02965C

23. Saratale GD, Bhosale R, Shobana S, Banu JR, Pugazhendhi A, Mahmoud $\mathrm{E}$, et al. A review on valorization of spent coffee grounds (SCG) 
towards biopolymers and biocatalysts production. Bioresour Technol. (2020) 314:123800. doi: 10.1016/j.biortech.2020.123800

24. Miao L, Zhou YH, Zhang HD, Hao ZB. Comparison of analytical methods for the quantitation of xylan in sugarcane bagasse. Food Sci. (2016) 37:162-7. doi: 10.7506/spkx1002-6630-201616026

25. Wang X, Zhang Z, Zhao M. Carboxymethylation of polysaccharides from Tremella fuciformis for antioxidant and moisture-preserving activities. Int J Biol Macromol. (2015) 72:526-30. doi: 10.1016/j.ijbiomac.2014. 08.045

26. Dubois M, Gilles KA, Hamilton JK, Colorimetric method for determination of sugars and related substances. Anal Chem. (1956) 28:350-6. doi: 10.1021/ac60111a017

27. Kintner PK, Van Buren JP. Carbohydrate interference and its correction in pectin analysis using the m-hydroxydiphenyl method. Food Sci. (1982) 47:756-9. doi: 10.1111/j.1365-2621.1982.tb12708.x

28. MM B. A rapid and sensitive method for the quantitation of microgram quantities of protein utilizing the principle of protein-dye binding. Anal Biochem. (1976) 72:248-54. doi: 10.1016/0003-2697(76)90527-3

29. Li J, Shang WT, Si X, Bu DD, Strappe P, Zhou ZK, et al. Carboxymethylation of corn bran polysaccharide and its bioactive property. Int J Food Sci Technol. (2017) 52:1176-84. doi: 10.1111/ijfs.13382

30. Hur SJ, Lim BO, Decker EA, McClements DJ. In vitro human digestion models for food applications. Food Chem. (2011) 125:1-12. doi: 10.1016/j.foodchem.2010.08.036

31. Minekus M, Alminger M, Alvito P, Ballance S, Bohn T, Bourlieu $\mathrm{C}$, et al. A standardised static in vitro digestion method suitable for food - an international consensus. Food Funct. (2014) 5:1113-24. doi: 10.1039/C3FO60702J

32. Zhao L, Qin Y, Guan R, Zheng W, Liu J, Zhao J. Digestibility of fucosylated glycosaminoglycan from sea cucumber and its effects on digestive enzymes under simulated salivary and gastrointestinal conditions. Carbohydr Polym. (2018) 186:217-25. doi: 10.1016/j.carbpol.2018.01.029

33. Wang Z-J, Xie J-H, Shen M-Y, Tang W, Wang H, Nie S-P, et al. Carboxymethylation of polysaccharide from Cyclocarya paliurus and their characterization and antioxidant properties evaluation. Carbohydr Polym. (2016) 136:988-94. doi: 10.1016/j.carbpol.2015.10.017

34. Geng WH, Venditti RA, Pawlak JJ, Chang HM, Pal L, Ford E. Carboxymethylation of hemicellulose isolated from poplar (Populus grandidentata) and its potential in water-soluble oxygen barrier films. Cellulose. (2020) 27:3359-77. doi: 10.1007/s10570-020-02993-2

35. Chen T, Liu H, Liu J, Li J, An Y, Zhu M, et al. Carboxymethylation of polysaccharide isolated from Alkaline Peroxide Mechanical Pulping (APMP) waste liquor and its bioactivity. Int J Biol Macromol. (2021) 181:211-20. doi: 10.1016/j.ijbiomac.2021.03.125

36. Cheng XQ, Li K, Chen XM, Jiang XN, Gai Y. Comparison of pectin structural monosaccharides in cell wall of dicotyledon and monocotyledon. J Beijing For Univ. (2012) 34:44-9. doi: 10.1007/s11783-011-0280-z

37. Kong F, Singh RP. Disintegration of solid foods in human stomach. J Food Sci. (2008) 73:R67-80. doi: 10.1111/j.1750-3841.2008.00766.x

38. Ohno N, Miura NN, Chiba N, Adachi Y, Yadomae T. Comparison of the immunopharmacological activities of triple and single-helical schizophyllan in mice. Biol Pharm Bull. (1995) 18:1242-7. doi: 10.1248/bpb.18.1242

39. Huang S, Chen F, Cheng H, Huang G. Modification and application of polysaccharide from traditional Chinese medicine such as Dendrobium officinale. Int J Biol Macromol. (2020) 157:385-93. doi: 10.1016/j.ijbiomac.2020.04.141

40. Qi X, Su T, Zhang M, Tong X, Pan W, Zeng Q, et al. Sustainable, flexible and biocompatible hydrogels derived from microbial polysaccharides with tailorable structures for tissue engineering. Carbohydr Polym. (2020) 237:116160. doi: 10.1016/j.carbpol.2020.116160

41. Shakhmatov EG, Toukach PV, Makarova EN. Structural studies of the pectic polysaccharide from fruits of Punica granatum. Carbohydr Polym. (2020) 235:115978. doi: 10.1016/j.carbpol.2020.1 15978
42. Velkova N, Doliska A, Zemljic LF, Vesel A, Saake B, Strnad S. Influence of carboxymethylation on the surface physical-chemical properties of glucuronoxylan and arabinoxylan films. Polym Eng Sci. (2015) 55:2706-13. doi: 10.1002/pen.24059

43. Martinez-Gonzalez AI, Diaz-Sanchez AG, de la Rosa LA, Vargas-Requena CL, Bustos-Jaimes I, Alvarez-Parrilla E. Polyphenolic compounds and digestive enzymes: in vitro non-covalent interactions. Molecules. (2017) 22:669. doi: 10.3390/molecules22040669

44. Liang L, Liu G, Zhang F, Li Q, Linhardt RJ. Digestibility of squash polysaccharide under simulated salivary, gastric and intestinal conditions and its impact on short-chain fatty acid production in type-2 diabetic rats. Carbohydr Polym. (2020) 235:115904. doi: 10.1016/j.carbpol.2020.115904

45. Kalantzi L, Goumas K, Kalioras V, Abrahamsson B, Dressman JB, Reppas C. Characterization of the human upper gastrointestinal contents under conditions simulating bioavailability/bioequivalence studies. Pharm Res. (2006) 23:165-76. doi: 10.1007/s11095-005-8476-1

46. Maldonado-Valderrama J, Wilde P, Macierzanka A, Mackie A. The role of bile salts in digestion. Adv Colloid and Interface Sci. (2011) 165:36-46. doi: 10.1016/j.cis.2010.12.002

47. Bauer E, Jakob S, Mosenthin R. Principles of physiology of lipid digestion. Asian-Australas J Anim Sci. (2005) 18:282-95. doi: 10.5713/ajas.2005.282

48. Harries JT, Sladen GJ. The effects of different bile salts on the absorption of fluid, electrolytes, and monosaccharides in the small intestine of the rat in vivo. Gut. (1972) 13:596-603. doi: 10.1136/gut.13.8.596

49. Ji NQ, Chen XD, Wang H, Ren C, Bao ZJ. Resistant mechanism of polymyxin resistant-Klebsiella pneumoniae. Chin J Antibiot. (2018) 43:1443-8. doi: 10.13461/j.cnki.cja.006438

50. Kim JH, Baek J, Sa S, Park J, Kih M, Kim W. Kestose-enriched fructo-oligosaccharide alleviates atopic dermatitis by modulating the gut microbiome and immune response. J Funct Foods. (2021) 85:104650. doi: 10.1016/j.jff.2021.104650

51. Rolfe MD, Rice CJ, Lucchini S, Pin C, Thompson A, Cameron ADS, et al. Lag phase is a distinct growth phase that prepares bacteria for exponential growth and involves transient metal accumulation. J Bacteriol. (2012) 194:686-701. doi: 10.1128/JB.06112-11

52. Cockburn DW, Koropatkin NM. Polysaccharide degradation by the intestinal microbiota and its influence on human health and disease. J Mol Biol. (2016) 428:3230-52. doi: 10.1016/j.jmb.2016.06.021

53. Bello B, Mustafa S, Tan JS, Ibrahim TAT, Tam YJ, Ariff AB, et al. Evaluation of the effect of soluble polysaccharides of palm kernel cake as a potential prebiotic on the growth of probiotics. 3 Biotech. (2018) 8:346. doi: 10.1007/s13205-018-1362-4

54. Feng R, Kou J, Chen S, Wang N, Wang W, Wang L, et al. Preparation optimization, characterization, and antioxidant and prebiotic activities of carboxymethylated polysaccharides from jujube. J Food Qual. (2021) 2021:3268149. doi: 10.1155/2021/3268149

Conflict of Interest: The authors declare that the research was conducted in the absence of any commercial or financial relationships that could be construed as a potential conflict of interest.

Publisher's Note: All claims expressed in this article are solely those of the authors and do not necessarily represent those of their affiliated organizations, or those of the publisher, the editors and the reviewers. Any product that may be evaluated in this article, or claim that may be made by its manufacturer, is not guaranteed or endorsed by the publisher.

Copyright $(2021$ Sun, Guan, Khoo and Li. This is an open-access article distributed under the terms of the Creative Commons Attribution License (CC BY). The use, distribution or reproduction in other forums is permitted, provided the original author(s) and the copyright owner(s) are credited and that the original publication in this journal is cited, in accordance with accepted academic practice. No use, distribution or reproduction is permitted which does not comply with these terms. 